\title{
Remote monitoring is high-tech, but is it humane?
}

Dr. John Hodges believed he had the ultimate animal monitoring system for his research. Using a commercially available blood glucose sensor that was surgically implanted into a rat's artery, he was able to monitor and record the rat's blood glucose concentration, activity and body temperature via telemetry for three weeks. The rat was placed on a platform that acted as electrocardiogram (ECG) electrodes so that he could measure the animal's heart rate and ECG. A remotely controlled infusion pump allowed him to provide specific amounts of subcutaneously administered insulin, and a small camera took a picture of the rat every 60 seconds. Although the system was already quite sophisticated, Hodges took his methodology to an even higher level by adapting the entire system to interface with his smart phone, allowing him to monitor and infuse his animals from home or any other location.

Hodges knew that his system worked because he had developed and tested it at his former institution with IACUC approval and an in-house grant. Now, at Great Eastern University, his goal was to monitor rats' blood glucose, activity, food intake and ECG response to dietary manipulations and new drugs being developed to treat type 2 diabetes. The Great Eastern IACUC was supportive of the study's scientific goals but questioned aspects of the methods to be used. Hodges' protocol clearly stated how critical it was to have close and frequent monitoring of his animals during the first 48 hours of their treatment with a new antidiabetic drug. After regular work hours, Hodges proposed to continue to monitor the animals once an hour from home. From there, he could provide insulin in the event that a rat experienced hyperglycemia (high blood sugar). However, the IACUC was equally concerned about hypoglycemia (low blood sugar) and other acute problems such as rapid or slow heart rate, cardiac arrhythmia or very high or low body temperature that might occur during the first 48 hours. Hodges responded that any animal that developed arrhythmia, severe hypoglycemia or an excessively high or low body temperature would be excluded from the study. This response did not satisfy the IACUC as it appeared to the committee that exclusion from the study, as proposed by Hodges, was not a humane endpoint and could result in unnecessary animal suffering until Hodges euthanized or provided treatment to the affected rats.

Hodges ran a one-man operation; he was young assistant professor with no laboratory support staff and very limited funds. Should the IACUC reconsider its position and allow Hodges to continue his study, or should it hold its ground? Should Hodges simply forget his elaborate at-home monitoring mechanism and remain with his animals, making observations every hour, for 48 hours? Is there a reasonable way to resolve this problem?

\section{RESPONSE}

\section{Reconsider, communicate, collaborate}

\author{
Lois Zitzow, MS, DVM, DACLAM \& \\ George Langan, DVM, DACLAM
}

According to the Guide for the Care and Use of Laboratory Animals, the IACUC should carefully consider two types of endpoints during protocol review: the experimental endpoint, which occurs when the scientific aims and objectives have been reached, and the humane endpoint, which occurs when pain or distress in an experimental animal is prevented, terminated or relieved ${ }^{1}$. In this scenario, Hodges has developed and validated a sophisticated monitoring system for his research study that will allow him to monitor and track changes in a number of physiologic parameters remotely.
Most of this monitoring appears to have been designed to assure that his animals can reach the experimental endpoint. However, the monitoring plan described in his protocol does not take advantage of data that could be used to determine early euthanasia criteria, such as the rats' appearance in the images he is collecting. In addition, by indicating that animals with severe hypoglycemia or excessively high or low body temperature would simply be excluded from the study rather than treated or humanely euthanized, Hodges has failed to identify humane endpoints.

The IACUC seems to be asking Hodges to define humane endpoints on the basis of abnormalities in the physiologic data, even if some of these abnormalities do not always indicate pain or distress. For example, bradycardia, tachycardia and cardiac arrhythmia can be benign or clinically insignificant, and mild or moderate hypoglycemia is often subclinical. Taken by themselves, these parameters might not be indicators that an animal requires treatment or has reached a humane endpoint.

The IACUC should reconsider this study. Hodges does not seem to understand the difference between humane and experimental endpoints, why they are important and how they relate to the study. The IACUC should take this opportunity to educate him about these concepts. In addition, the IACUC, the veterinarians and Hodges should work together to assess the types of data being collected, determine which data are clinically relevant and develop better humane endpoints. The IACUC should also encourage the use of the remote monitoring system because it will enable more aggressive monitoring, thus addressing the animal welfare concerns, while allowing the investigator to conduct this work with limited staff and funds. This is especially important in today's funding climate. Furthermore, the 